\title{
Tunable light trapping and absorption enhancement with graphene ring arrays
}

\author{
Shuyuan Xiao, ${ }^{a}$ Tao Wang, ${ }^{* a}$ Yuebo Liu, ${ }^{b}$ Chen Xu, ${ }^{c}$ Xu Han, ${ }^{a}$ and Xicheng Yan $^{a}$
}

\author{
Received Xth $X X X X X X X X X X 20 X X$, Accepted Xth $X X X X X X X X X 20 X X$ \\ First published on the web Xth $X X X X X X X X X X 200 X$ \\ DOI: $10.1039 / b 000000 x$
}

Surface plasmon resonance (SPR) has been intensively studied and widely employed for light trapping and absorption enhancement. In the mid-infrared and terahertz (THz) regime, graphene supports the tunable SPR via manipulating its Fermi energy , and enhances light-matter interaction at the selective wavelength. In this work, periodic arrays of graphene rings are proposed to introduce tunable light trapping with good angle polarization tolerance and enhance the absorption in the light-absorbing materials nearby to more than one order. Moreover, the design principle here could be set as a template to achieve multi-band plasmonic absorption enhancement by introducing more graphene concentric rings into each unit cell. This work not only opens , up new ways of employing graphene SPR, but also leads to practical applications in high-performance simultaneous multi-color 'photodetection with high efficiency and tunable spectral selectivity.

\section{Introduction}

In recent years, the light absorption has demonstrated a variety of optoelectronic applications in photovoltaic cells $\frac{1-3}{2}$, spatial light modulation ${ }^{4,5}$, ultrasensitive biosensing ${ }^{6-9}$, photocatalytic reaction ${ }^{10-13}$ and photodetection ${ }^{14-16}$. The extinction of the light-absorbing semiconductors employed in these devices is expected to be high enough to guarantee sufficient light absorption. On the other hand, there is also urgent need to reduce the thickness and volume of them to decrease the resource consumption, reduce the material deposition require'ment, and improve the integrability, which also inevitably weaken the interaction between light and the semiconduc"tors ${ }^{17,18}$. Moreover, the emergency of two-dimensional (2D) materials with unique properties has stimulated great interests in scientific studies and technological developments ${ }^{19-24}$, but the innate atomic thickness greatly hampers the utilization of light 25,26 . As a result, enhancing the light absorption in the above-mentioned semiconductors and 2D materials to satisfy 'the requirements of practical applications, especially of some light-driven-related applications such as photodetection, has become of increasing importance. Surface plasmonic resonance (SPR), the collective electronic excitation at the interface between a metal and a dielectric medium, has been introduced as one of the most widely used approaches to en-

${ }^{a}$ Wuhan National Laboratory for Optoelectronics, Huazhong University of Science and Technology, Wuhan 430074, People's Republic of China. E-mail: wangtao@hust.edu.cn

${ }^{b}$ School of Information and Optoelectronic Science and Engineering, South China Normal University, Guangzhou 510006, People's Republic of China

${ }^{c}$ Department of Physics, New Mexico State University, Las Cruces 88001, United State of America hance the light absorption for it can strongly couple to the incidence light and lead to efficient light trapping and absorption enhancement in the subwavelength scale structure ${ }^{27-30}$. Based on this, a range of plasmonic structures such as metal strip $^{31-33}$, disk ${ }^{34,35}$, ring ${ }^{36,37}$, cross $^{38}$ and other shapes 39,40 have been proposed to integrate with the light-absorbing materials to improve their absorption performances. However, the spectral responses of these metal SPRs are dominated by the geometric parameters, and the operation wavelength will be unchangeable once these devices are manufactured, which still restricts flexible applications in practice.

Graphene is a monolayer carbon material that has become a promising building block in the state of the art technology due to its remarkable mechanical, thermal and electromagnetic properties ${ }^{41-44}$. As a typical example of 2D materials, the interaction between light and graphene is quite weak and a single sheet of graphene can absorb only $2.3 \%$ energy of the incidence light in the visible and near infrared. Unlike other 2D materials, graphene itself behaves like metals when coupling with the incidence light and supports SPR in the midinfrared and terahertz $(\mathrm{THz})$ region, therefore benefits to light trapping and absorption enhancement ${ }^{45-47}$. Moreover, compared with metal structure, the continuously tunable surface conductivity of graphene via manipulating its Fermi energy by electric gating or chemical doping enable dynamically tunable resonance ${ }^{48-52}$, which can be used to amplify the photoresponse to the incidence light at the selective wavelength, making it possible for highly accurate photodetection ${ }^{53}$. Tunable single- $-\frac{54}{}$, dual- -55 and multi-band 56 absorption enhancement with graphene plasmonics have been realized and even perfect absorption also achieved with graphene absorber backed 
by a gold mirror $57-59$. However, most of these previous works concentrated on the absorption enhancement in graphene itself while little attention has been paid to trap light and enhance the light absorption in other light-absorbing materials with graphene SPR.

To demonstrate this kind of use of graphene SPR, a simulation study on tunable light trapping and absorption enhancement has been systematically conducted in this work with a hybrid periodic array composed of a graphene ring on the top of the light-absorbing materials (thin film semiconductor or 2D material) separated by an insulating layer. Graphene ring shows good operation angle polarization tolerance due to its high symmetry, and it possesses the advantage of more free geometric parameters compared with disk $\underline{60,61}$. The simulation results show that the excitations of SPR in the graphene ring trap a sizable part of the incidence light and significantly enhance the light absorption in surrounding absorbing materials. With manipulating the Fermi energy of graphene and adjusting the geometric parameters, the absorption could be modulated over a large range. In addition, our proposed structure leaves room for introducing more graphene rings into the unit cell to form multiple resonating structure, and consequently enables dynamically tunable multi-band absorption enhancement in the below light-absorbing materials, which is considered to play a significant role in the simultaneous multi-color photodetection with high efficiency and tunable spectral selectivity.

\section{The geometric structure and numerical model}

The schematic geometry of our proposed structure is shown in Figure 1. The unit cell is arranged in a periodic array with a lattice constant $P=300 \mathrm{~nm}$ and composed of a graphene ring on the top of the absorbing layer separated by an insulating layer. $R=75 \mathrm{~nm}$ is the radius of the graphene ring with a width $W=30 \mathrm{~nm}$ and the effective thickness is set as $t_{g}=1$ $\mathrm{nm}$. The thicknesses of the insulating layer and the absorbing layer are $t_{i}=20 \mathrm{~nm}$ and $t_{a}=100 \mathrm{~nm}$, and the substrate is assumed to be semi-infinite. Both the insulating layer and the substrate are considered as lossless dielectric with a real permittivity of $\varepsilon_{d}=1.96$. Comparable with some typical materials employed for photodetection in the mid-infrared and $\mathrm{THz}$ regime such as $\mathrm{HgCdTe}(\mathrm{MCT}) \frac{62}{2}$, the absorbing layer is modeled as thin film semiconductor through a complex permitivity of $\varepsilon_{a}=\varepsilon^{\prime}+i \varepsilon^{\prime \prime}$, where $\varepsilon^{\prime}=10.9$ and $\varepsilon^{\prime \prime}$ is related to the attenuation coefficient $\alpha=-(2 \pi / \lambda) \operatorname{Im}\left(\sqrt{\varepsilon^{\prime}+i \varepsilon^{\prime \prime}}\right)$ accounting for the loss.

Graphene is considered as a 2D material and modeled by the surface conductivity $\sigma_{g}$ with the random-phase approximation (RPA) in the local limit, including the intraband and interband

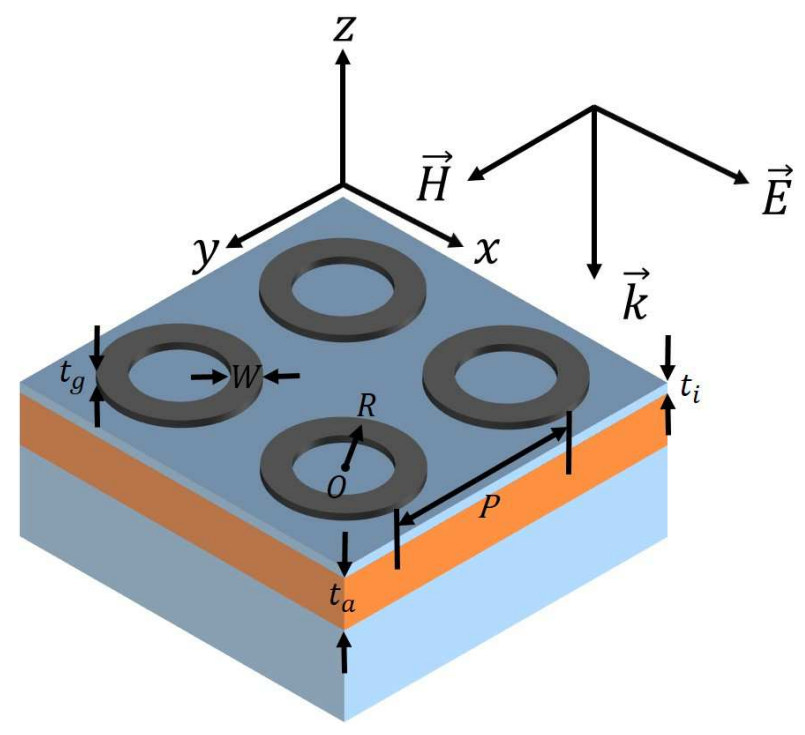

Fig. 1 The schematic geometry of our proposed hybrid periodic array. Each unit cell is composed of a graphene ring on the top of the absorbing layer separated by an insulating layer.

transitions 63,64

$$
\begin{aligned}
\sigma_{g} & =\sigma_{\text {intra }}+\sigma_{\text {inter }} \\
& =\frac{2 e^{2} k_{B} T}{\pi \hbar^{2}} \frac{i}{\omega+i \tau^{-1}} \ln \left[2 \cosh \left(\frac{E_{F}}{2 k_{B} T}\right)\right] \\
& +\frac{e^{2}}{4 \hbar}\left[\frac{1}{2}+\frac{1}{\pi} \arctan \left(\frac{\hbar \omega-2 E_{F}}{2 k_{B} T}\right)-\frac{i}{2 \pi} \ln \frac{\left(\hbar \omega+2 E_{F}\right)^{2}}{\left(\hbar \omega-2 E_{F}\right)^{2}+4\left(k_{B} T\right)^{2}}\right]
\end{aligned}
$$

where $e$ is the charge of an electron, $k_{B}$ is the Boltzmann constant, $T$ is the operation temperature, $\hbar$ is the reduced Planck's constant, $\omega$ is the angular frequency of the incidence light, $\tau$ is the relaxation time and $E_{F}$ is the Fermi energy. In the lower $\mathrm{THz}$ regime, the interband contributions can be safely neglected due to the Pauli exclusion principle and therefore the surface conductivity can be approximated to a Drude model ${ }^{65}$

$$
\sigma_{g}=\frac{e^{2} E_{F}}{\pi \hbar^{2}} \frac{i}{\omega+i \tau^{-1}}
$$

herein the relaxation time $\tau=\left(\mu E_{F}\right) /\left(e v_{F}^{2}\right)$ depends on the the electron mobility $\mu \approx 10000 \mathrm{~cm}^{2} / \mathrm{Vs}$, the Fermi energy $E_{F}$ and the Fermi velocity $v_{F} \approx 10^{6} \mathrm{~m} / \mathrm{s}$. Furthermore, the permitivity of graphene can be obtained by $\underline{49.66}$

$$
\varepsilon_{g}=1+\frac{i \sigma_{g}}{\varepsilon_{0} \omega t_{g}}
$$

where $\varepsilon_{0}$ is the permittivity of vacuum.

As predicted in the equation (2), the surface conductivity of graphene can be tuned via manipulating its Fermi energy. 
The optical properties of graphene and thus the performances of light trapping in our proposed structure can be dynamically tuned, which can not be realized with metal SPR devices. It is also noted that the most conventional way to manipulate the Fermi energy of graphene, i.e., the backgate configuration would introduce additional electrodes, which makes the fabrication process of the hybrid periodic array much more complicated and is not conducive to practical application. Therefore, in Figure 2 another two feasible methods of tuning $E_{F}$ are presented: (a) through a uniform external electric field generated by either distant gates or low-frequency radiation 67.68 and (b) through the $\mathrm{HNO}_{3}$ vapor made by external heater $\stackrel{69,70}{ }$.

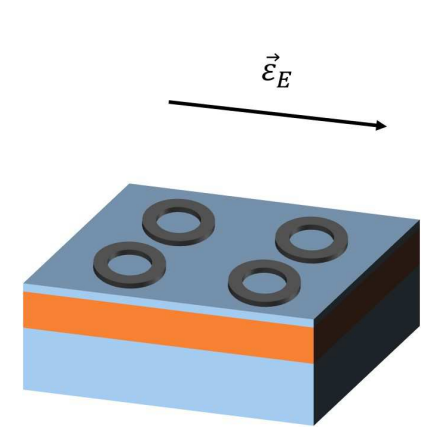

(a)

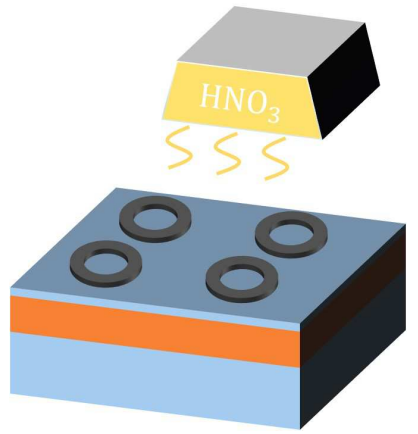

(b)
Fig. 2 Two feasible methods of tuning $E_{F}$ : (a) through a uniform external electric field $\vec{\varepsilon}_{E}$ and (b) through the $\mathrm{HNO}_{3}$ vapor.

In the initial setup, the attenuation coefficient of the absorbing layer $\alpha=-0.1 \mu^{-1}$ and the Fermi energy of graphene $E_{F}=0.6 \mathrm{eV}$ are considered, and the influences of them will be analyzed later. The numerical simulations are conducted using the finite-difference time-domain method with the commercial software FDTD Solutions. The anti-symmetric and symmetric boundary conditions are respectively employed in the $x$ and $y$ directions throughout the calculations except when studying the angle polarization tolerance, and perfectly matched layers are utilized in the $z$ direction along the propagation of the incidence plane wave.

\section{Simulation results and discussions}

For the above structure, a typical resonance response to normal incidence light with transmission suppression and absorption enhancement is displayed at around $19.6 \mu \mathrm{m}$ in Figure 3. The total absorption of this structure is $A=30.8 \%$ and the absorption in the absorbing layer is $A^{\prime}=17.4 \%$. Now that the absorbing layer is set to $100 \mathrm{~nm}$ thick with the attenuation coefficient $\alpha=-0.1 \mu \mathrm{m}^{-1}$, which corresponds to a weak absorption of $2 \%$ in the impedance matched media, about 8.7

times absorption enhancement in the absorbing layer has been realized at the resonance.

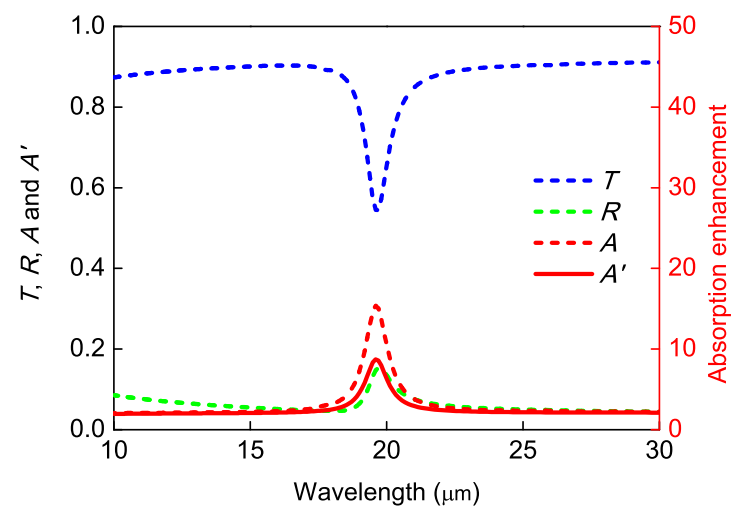

Fig. 3 The simulated transmission $T$, reflection $R$ and absorption $A$ as well as the absorption in the absorbing layer $A^{\prime}$ with the attenuation coefficient $\alpha=-0.1 \mu \mathrm{m}^{-1}$ and the Fermi energy of graphene $E_{F}=0.6 \mathrm{eV}$. The enhancement factor of absorption in the absorbing layer is also shown compared to that in the impedance matched media.

It is not difficult to believe the absorption enhancement should be ascribed to graphene SPR. The localized collective electronic excitations strongly couple to and trap the incidence light and enhance the absorption in the nearby absorbing layer. To better understand and make use of the absorption band, the electric field distributions at the resonance are plotted below. As shown in Figure 4(a), the $x-y$ plane electric field distribu-

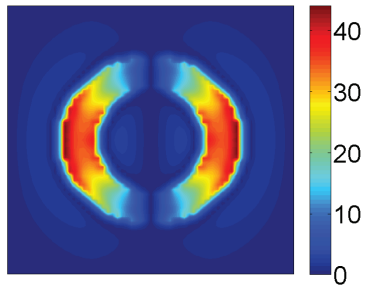

(a)

Fig. 4 The simulated electric field distributions (a) in the $x-y$ plane $\left(\left|E_{z}\right|\right)$ and (b) in the $x-z$ cross plane $\left(\left|E_{y}\right|\right)$ at the resonance with the attenuation coefficient $\alpha=-0.1 \mu \mathrm{m}^{-1}$ and the Fermi energy of graphene $E_{F}=0.6 \mathrm{eV}$.

tion $\left(\left|E_{z}\right|\right)$ at the resonance shows characteristic behavior of the electric dipole and is symmetric to the $y$-axis due to the $x$-polarization of the incidence light. Meanwhile, the $x-z$ cross plane electric field distribution $\left(\left|E_{y}\right|\right)$ in Figure 4(b) clearly represents that the plasmon-induced strong field confinement 
extends to the absorbing layer and enhances the light-matter interaction.

Theoretically, it has been known that that the effective wavelength of the above-mentioned dipole resonance is approximately equal to the circumference of the corresponding ring

$$
\lambda_{e f f}=2 \pi R,
$$

and thus the resonance wavelength can be predicted from

$$
\lambda_{\text {res }}=\lambda_{e f f} n_{e f f}=2 \pi R n_{e f f}
$$

where $n_{\text {eff }}$ is the effective refractive index of the ring waveguide. For a graphene ribbon waveguide with the same width $(W=30 \mathrm{~nm}), n_{\text {eff }}$ at difderent Fermi energies ranging from $0.4 \mathrm{eV}-1.2 \mathrm{eV}$ are plotted in Figure 5 with FDTD Solutions. It can be seen that $n_{e f f}$ is a function of $E_{F}$, meaning that the

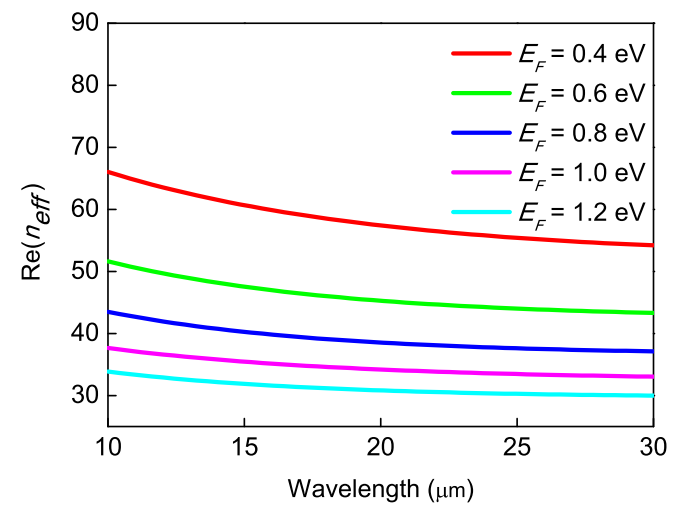

Fig. 5 The calculated $n_{\text {eff }}$ with the attenuation coefficient $\alpha=-0.1 \mu \mathrm{m}^{-1}$ and the Fermi energy of graphene $E_{F}$ ranging from 0.4 to $1.2 \mathrm{eV}$.

resonance wavelength of our proposed structure can be expediently shifted via manipulating the Fermi energy of graphene, which lays the direct foundation for the light trapping and absorption enhancement in the absorbing layer at the selective wavelength.

As the only two lossy media within the hybrid structure, there unavoidably exists a competition of absorption between graphene and the absorbing layer nearby. The attenuation coefficient of the low light-absorbing efficiency material, initially set to $\alpha=-0.1 \mu \mathrm{m}^{-1}$, plays a dominant role in the distribution of light absorption. The total absorption $A$ and the absorption in the absorbing layer $A^{\prime}$ are also simulated with different $\alpha$ in Figure 6. When $\alpha=-0.05 \mu \mathrm{m}^{-1}$, corresponding to a poor absorption of $1 \%$ in the impedance matched media, the total absorptions increase to $34.6 \%$ and the absolute absorption in the absorbing layer is $A^{\prime}=13.4 \%$ with more than one order enhancement at the resonance due to the lower absorption loss of the total structure and the greater quality factor of the resonance. When $\alpha=-0.2 \mu \mathrm{m}^{-1}$, corresponding to about $4 \%$ absorption in the impedance matched media, the total absorptions decrease to $A=27.0 \%$ while the absorption in the absorbing layer is $A^{\prime}=20.0 \%$, in which case about five times enhancement is achieved. It is also noteworthy that absorption band stays at nearly the same resonance wavelength in that the variations in the attenuation coefficient $\alpha$ here are not big enough to bring about visible influences on $n_{e f f}$.

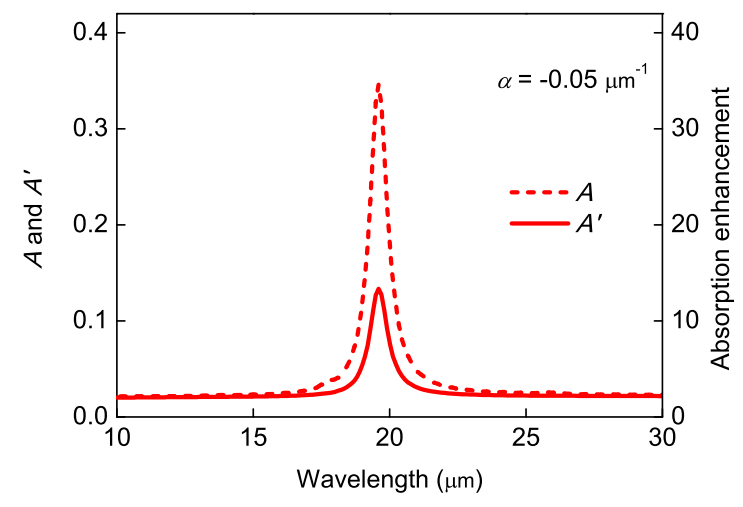

(a)

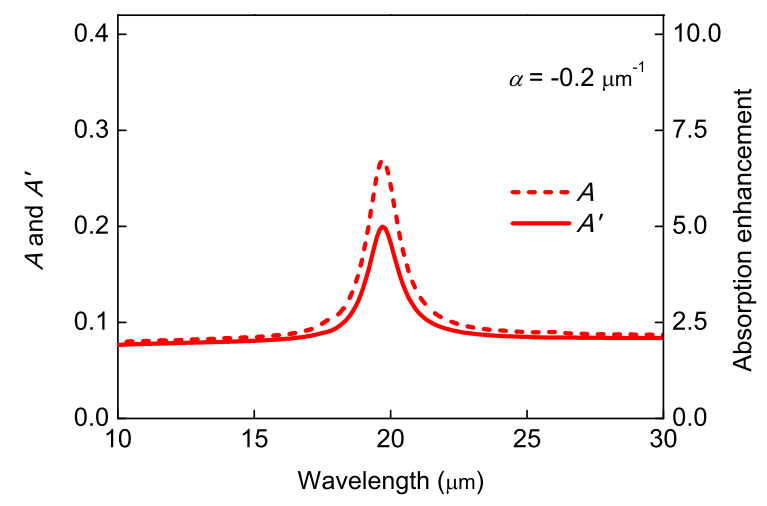

(b)

Fig. 6 The simulated absorption $A$ and the absorption in the absorbing layer $A^{\prime}$ with the attenuation coefficient (a) $\alpha=-0.05$ $\mu \mathrm{m}^{-1}$ and (b) $\alpha=-0.2 \mu \mathrm{m}^{-1}$ and the Fermi energy of graphene $E_{F}=0.6 \mathrm{eV}$. The enhancement factor of absorption in the absorbing layer is also shown compared to that in the impedance matched media.

To analyze the tunable property of the light trapping, the absorption in the absorbing layer with various Fermi energy of graphene has been simulated in Figure 7. When the Fermi energy starting at $0.4 \mathrm{eV}$, the resonance wavelength is 24.0 
$\mu \mathrm{m}$ and the absorption is $11.3 \%$. As $E_{F}$ increases to $0.8 \mathrm{eV}$, the resonance shifts to $17.1 \mu \mathrm{m}$ and the absorption goes up to $22.1 \%$ with more than one order absorption enhancement. Finally while $E_{F}$ comes to $1.2 \mathrm{eV}$, the resonance absorption reaches as high as $27.7 \%$ at $13.8 \mu \mathrm{m}$. The physical mechanism lies in that the conductivity of graphene increases with $E_{F}$ and thus graphene SPR becomes less lossy, which leads to more absorption in the absorbing layer. The resonance wave-

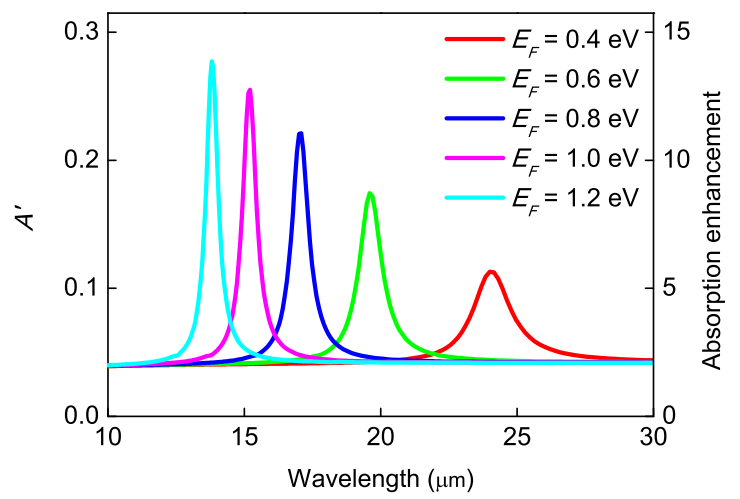

Fig. 7 The simulated absorption in the absorbing layer $A^{\prime}$ with the attenuation coefficient $\alpha=-0.1 \mu \mathrm{m}^{-1}$ and the Fermi energy of graphene $E_{F}$ ranging from 0.4 to $1.2 \mathrm{eV}$. The enhancement factor of absorption in the absorbing layer is also shown compared to that in the impedance matched media.

lengths in theoretical analysis (red line) and simulation (green line) are plotted in Figure 8. One can see the theoretical resonance wavelengths at different Fermi energies, calculated with the equation (5), follow the fairly similar trend as the simulation results, though minute differences exist. This could be because the $n_{\text {eff }}$ was calculated in the graphene ribbon waveguide, which has some geometric differences with the ring waveguide in our proposed structure, and the deviation of $n_{\text {eff }}$ should lead to the deviation of the resonance wavelength. Considering this, the agreement between theory and simulation is reasonable and could be employed in predicting the resonance wavelength of our proposed device for light trapping and absorption enhancement. As a consequence, photoresponse amplification with high efficiency and tunable spectral selectivity can be achieved with graphene SPR for highly accurate photodetection, which can not be realized with metal SPR.

Considering the high symmetry of our proposed structure, the incidence angle and polarization dependence of graphene ring is expected to be weak. The light absorption properties at various angle for both TE and TM configurations have been investigated. As illustrated in Figure 9(a) and (b), the absorption in the absorbing layer keeps nearly the same for incidence an-

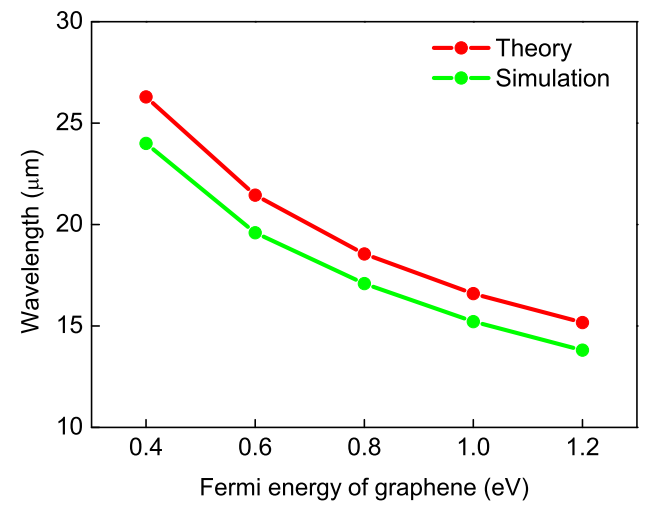

Fig. 8 The resonance wavelengths in theoretical analysis and simulation with the attenuation coefficient $\alpha=-0.1 \mu \mathrm{m}^{-1}$ and the Fermi energy of graphene $E_{F}$ ranging from 0.4 to $1.2 \mathrm{eV}$.

gle up to 45 degrees. On the other hand, the resonance wavelength is only determined by the radius and the Fermi energy of the graphene ring, indicated by the equation (5), and does not depend on the incidence angle and polarization, which is also reflected in the figures. Consequently, the good operation angle and polarization tolerance of graphene ring will benefit the practical applications in light trapping and absorption enhancement.

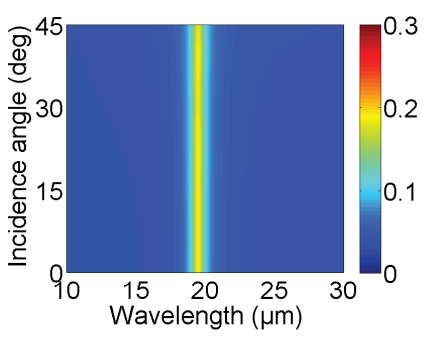

(a)

Fig. 9 The simulated angular dispersions of the absorption in the absorbing layer with the attenuation coefficient $\alpha=-0.1 \mu \mathrm{m}^{-1}$ and the Fermi energy of graphene $E_{F}=0.6 \mathrm{eV}$ for (a) TE and (b) TM configurations.

In the most recent years, 2D materials have emerged as promising building blocks in the state of the art optoelectronic devices among a very wide range of electromagnetic spectrum ${ }^{25}$. Compared with the traditional photonic materials, 2D materials exhibit a lot of exceptional properties. The quantum confinement in the direction perpendicular to the 2D plane leads to many novel optical and electronic properties ${ }^{21,71-73}$, and the naturally passivated surfaces make them easy to integrate with photonic structures such as waveguides $\frac{74.75}{}$ and 
cavities ${ }^{76,77}$. In addition to graphene, a variety of other $2 \mathrm{D}$ materials such as transition metal dichalcogenides have been employed in photodetection and other optoelectronic applications ${ }^{78,79}$. Though possessing very high quantum efficiency for light-matter interaction, the absorption of these 2D materials is insufficient in practical devices due to the innate thinness. Therefore, integration with SPR offers an effective solution for the absorption enhancement and further applications in photodetection. As shown in Figure 10, the $100 \mathrm{~nm}$ thick absorbing layer is replaced with 2D material, which is modelled as a $1 \mathrm{~nm}$ thick conductive film with an optical conductance $G_{0}=2.65 \times 10^{-5} \Omega^{-1}$, corresponding to an absorption of about $1 \%$ for a free standing film. Similar to that in the

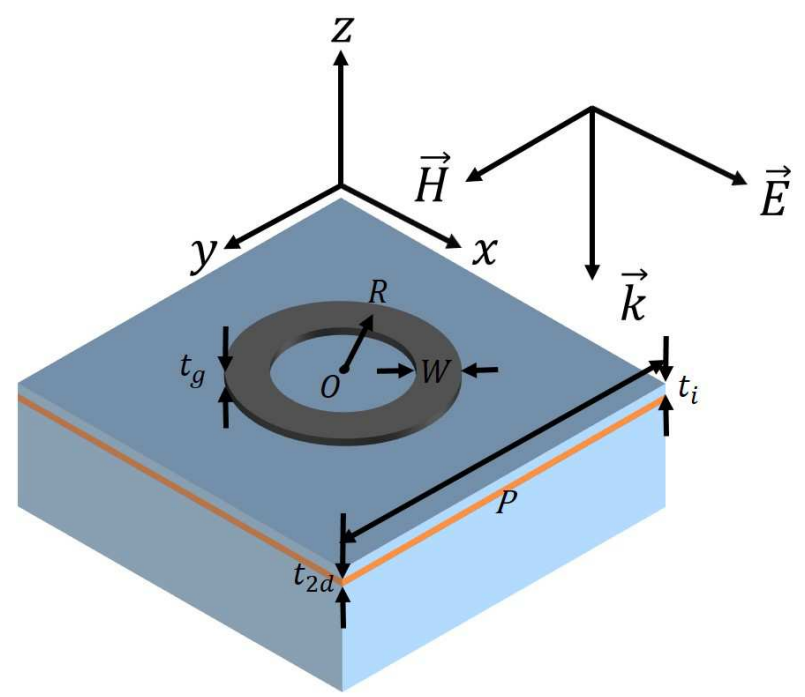

Fig. 10 The schematic geometry of the unit cell composed of a graphene ring on the top of the absorbing layer separated by an insulating layer and thin film semiconductor is replaced with $2 \mathrm{D}$ material.

thin film semiconductor, the resonance response is also found here. Figure 11 plots the absorption enhancement in the 2D absorbing layer while Fermi energy of graphene varies from $0.4 \mathrm{eV}$ to $1.2 \mathrm{eV}$ in step of $0.2 \mathrm{eV}$. When $E_{F}$ starting at 0.4 $\mathrm{eV}$, the resonance wavelength locates at $20.5 \mu \mathrm{m}$, and the absorption is $7.9 \%$ with an enhancement factor of 7.9. When $E_{F}$ increases to $0.6 \mathrm{eV}$, the resonance blue shifts to $16.6 \mu \mathrm{m}$ and the absorption goes up to $14.3 \%$ with more than one order absorption enhancement. And while $E_{F}$ comes to $1.2 \mathrm{eV}$, the absorption reaches as high as $25.2 \%$ at $11.7 \mu \mathrm{m}$ and the enhancement factor over 25 is obtained at the resonance.

In the above simulations, the geometric parameters of the hybrid structure are fixed and the tunability is mainly demonstrated via manipulating the Fermi energy of graphene. In fact, the light trapping and absorption enhancement could also be tuned with the geometric variations of the structure (See ESI

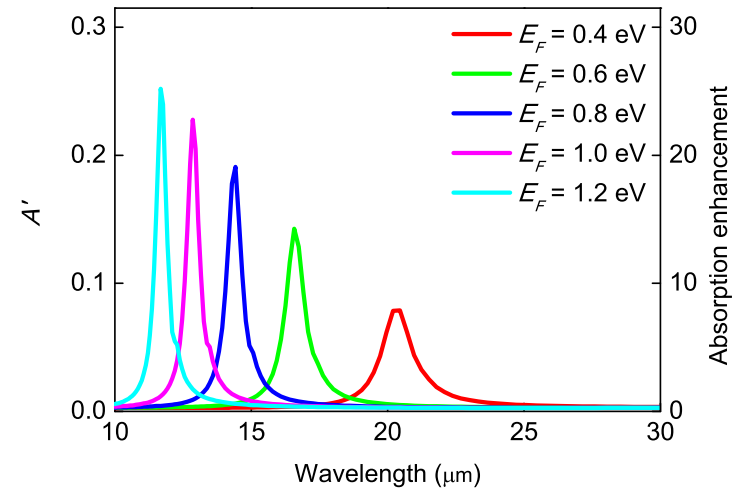

Fig. 11 The simulated absorption in the $2 \mathrm{D}$ material $A^{\prime}$ with an optical conductance $G_{0}=2.65 \times 10^{-5} \Omega^{-1}$ and the Fermi energy of graphene $E_{F}$ ranging from 0.4 to $1.2 \mathrm{eV}$. The enhancement factor of absorption in the absorbing layer is also shown compared to that in the impedance matched media.

Figure S1). It is also noted that the total thickness of the hybrid structure is much smaller than the wavelength of the incidence light, in which case the universal maximum absorption can be evaluated with regarding the whole structure as a thin film separating two different media (air with the refractive index of $n_{\text {air }}=1$ and the substrate with $n_{\text {sub }}=1.4$ ), i.e., $A_{\text {max }}=41.7 \%(=1 /(1+1.4))^{80}$. Perfect light absorption in the hybrid structure and further absorption enhancement in the absorbing layer are obtained with a gold mirror (See ESI Figure S2). Moreover, another major advantage of our proposed structure lies in that it is straightforward to include more rings into the unit cell to form a compact structure with multiple resonances. For example, the light trapping and absorption enhancement with a pair of graphene concentric rings are studied. Figure 12 depicts the schematic geometry of the structure composed of a pair of graphene concentric rings on the top of the thin film semiconductor separated by a insulating layer. The inner graphene ring remains as $R_{1}=R=75 \mathrm{~nm}$ and the outer one is added as $R_{2}=125 \mathrm{~nm}$, and the attenuation coefficient and other geometric parameters keep the same.

Figure 13 illustrates the absorption in the absorbing layer at different Fermi energies. The results show that another absorption band, as expected, appears at longer wavelength, and when the Fermi energy gets to $1.0 \mathrm{eV}$, the absorption goes up to $21.7 \%$ at $26.9 \mu \mathrm{m}$, which implies more than one order absorption enhancement is achieved simultaneously at both resonances. More interesting, for each absorption band at shorter wavelength, the absorption keeps nearly the same strength and the resonance wavelength stays nearly the same position as with single graphene ring, which suggest each resonance could be approximated to individual dipole response 


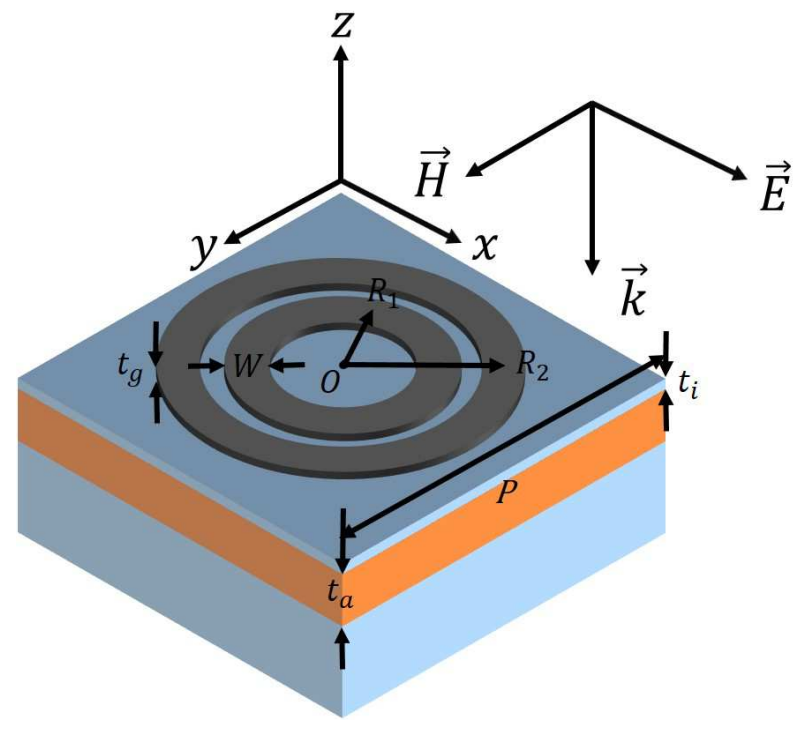

Fig. 12 The schematic geometry of the unit cell composed of a pair of graphene concentric rings on the top of the absorbing layer separated by an insulating layer.

of the corresponding graphene ring. To confirm this, the $x$ $y$ plane electric field distribution $\left(\left|E_{z}\right|\right)$ and the $x-z$ cross plane electric field distribution $\left(\left|E_{y}\right|\right)$ with the attenuation coefficient $\alpha=-0.1 \mu \mathrm{m}^{-1}$ and the Fermi energy of graphene $E_{F}=0.6$ $\mathrm{eV}$ at the resonances are presented in Figure 14. One can tell the electric field mainly concentrates in either inner or outer ring and the mutual couplings between them are rather weak. Therefore, the design principle here could be safely set as a template to achieve tunable multiband plasmonic absorption enhancement in the below light-absorbing materials, and consequently meets the urgent need for the simultaneous multicolor photodetection with high efficiency and tunable spectral selectivity.

\section{Conclusions}

To conclude, tunable light trapping and absorption enhancement have been numerically investigated in a hybrid periodic array composed of a graphene ring on the top of the absorbing layer separated by an insulating layer. It is found that the excitations of localized collective electrons in graphene strongly couple to and trap the incidence light in the near-field, and significantly enhance the absorption in the light-absorbing materials (thin film semiconductor or 2D material) to more than one order at the resonance. Compared with the traditional noble metal plasmonic devices, the absorption enhancement here can be dynamically tuned via manipulating the Fermi energy of graphene over such a large range, thus enables tunable photodetection and other optoelectronic applications in

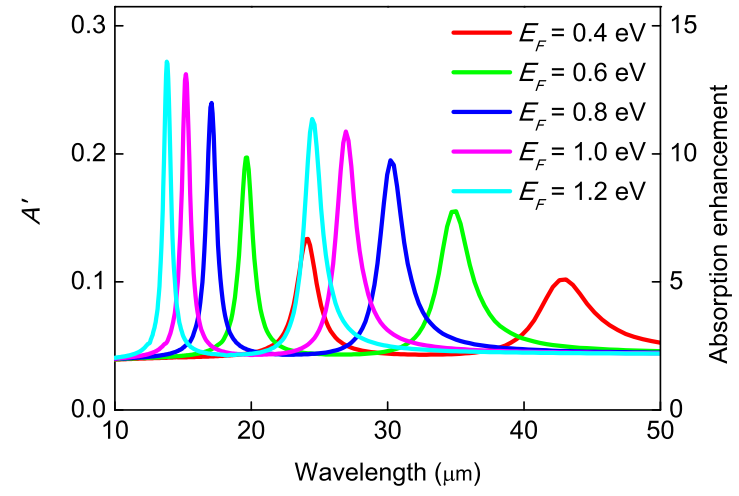

Fig. 13 The simulated absorption in the absorbing layer $A^{\prime}$ with the attenuation coefficient $\alpha=-0.1 \mu \mathrm{m}^{-1}$ and the Fermi energy of graphene $E_{F}$ ranging from 0.4 to $1.2 \mathrm{eV}$. The enhancement factor of absorption in the absorbing layer is also shown compared to that in the impedance matched media.
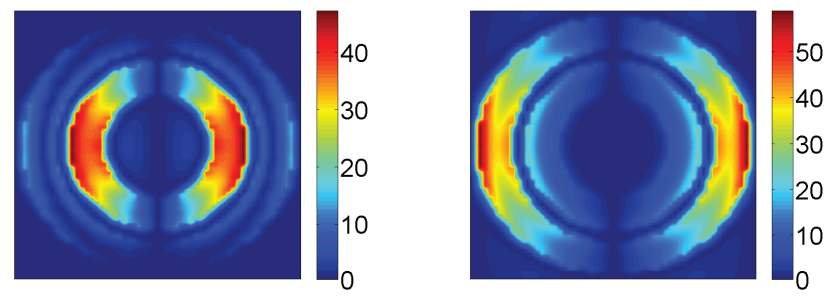

(a)

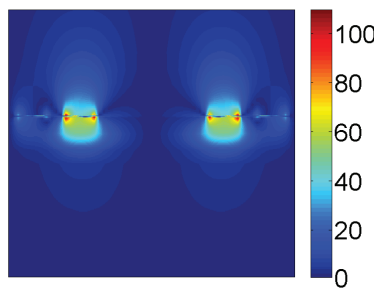

(c)

Fig. 14 The simulated electric field distributions (a)-(b) in the $x-y$ plane $\left(\left|E_{z}\right|\right)$ and (c)-(d) in the $x-z$ cross plane $\left(\left|E_{y}\right|\right)$ at the resonances with the attenuation coefficient $\alpha=-0.1 \mu \mathrm{m}^{-1}$ and the Fermi energy of graphene $E_{F}=0.6 \mathrm{eV}$. 
the mid-infrared and $\mathrm{THz}$ regime. As an extension, our proposed structure leaves room for introducing more graphene concentric rings to the unit cell to achieve multi-band absorption enhancement, therefore lays the foundation for simultaneous photodetection at multiple wavelengths with high efficiency and tunable spectral selectivity.

\section{Acknowledgments}

The author Shuyuan Xiao (SYXIAO) expresses his deepest gratitude to his $\mathrm{Ph} . \mathrm{D}$. advisor Tao Wang for providing guidance during this project. SYXIAO would also like to thank Prof. Jianfa Zhang (National University of Defense Technology) for his guidance to the modeling of light-absorbing materials and Dr. Qi Lin (Hunan Univerisity) for beneficial discussion on graphene optical properties. This work is supported by the National Natural Science Foundation of China (Grant No. 61376055 and 61006045), and the Fundamental Research Funds for the Central Universities (HUST: 2016YXMS024).

\section{References}

1 M.D. Kelzenberg, S. W. Boettcher, J. A. Petykiewicz, D. B. Turner-Evans, M. C. Putnam, E. L. Warren, J. M. Spurgeon, R. M. Briggs, N. S. Lewis and H. A. Atwater, Nat. Mater, 2010, 9, 239-244.

2 X. Huang, S. Han, W. Huang and X. Liu, Chem. Soc. Rev., 2013, 42, 173-201.

3 A. Dessi, M. Calamante, A. Mordini, M. Peruzzini, A. Sinicropi, R. Basosi, F. Fabrizi de Biani, M. Taddei, D. Colonna, Aldo di Carlode, G. Reginato and L. Zani, RSC Adv., 2015, 5, 32657-32668.

4 X. Liu, T. Starr, A. F. Starr and W. J. Padilla, Phys. Rev. Lett., 2010, 104, 207403.

5 S. Savo, D. Shrekenhamer and W. J. Padilla, Adv. Opt. Mater., 2014, 2 , 275-279.

6 K. Chen, R. Adato and H. Altug, Acs Nano, 2012, 6, 7998-8006.

7 B. X. Wang, X. Zhai, G. Z. Wang, W. Q. Huang and L. L. Wang, J. Appl. Phys. 2015, 117, 014504.

8 F. Chen, M. Lin, Y. Zhao and Y. Zhao, RSC Adv., 2016, 6, 15709-15715.

9 Q. Lin, X. Zhai, L. L. Wang, X. Luo, G. D. Liu, J. P. Liu and S. X. Xia, Appl. Phys. Express, 2016, 9, 062002.

10 X. Chen, L. Liu, Y. Y. Peter and S. S. Mao, Science, 2011, 331, 746-750.

11 F. F. Abdi, A. Dabirian, B. Dam and Roel van de Krol, Phys. Chem. Chem. Phys., 2014, 16, 15272-15277.

12 N. Zhou, V. Lopez-Puente, Q. Wang, L. Polavarapu, I. Pastoriza-Santos and Q. H. Xu, RSC Adv., 2015, 5, 29076-29097.

13 G. Wang, H. Chen, Y. Li, A. Kuang, H. Yuan and G. Wu, Phys. Chem. Chem. Phys., 2015, 17, 28743-28753.

14 M. W. Knight, H. Sobhani, P. Nordlander and N. J. Halas, Science, 2011, 332, 702-704.

15 H. Li, J. Yang, J. Zhang and M. Zhou, RSC Adv, 2012, 2, 6258-6261.

16 P. Sharmaa, R. Singha, V. Awasthia, S. K. Pandeyb, V. Garga and S. Mukherjee, RSC Adv., 2015, 5, 85523-85529.

17 D. M. Callahan, J. N. Munday and H. A. Atwater, Nano lett., 2012, 12, 214-218.

18 J. T. Liu, X. H. Deng, W. Yang and J. Li, Phys. Chem. Chem. Phys., 2015, 17, 3303-3308.

19 F. Bonaccorso, Z. Sun, T. Hasan, and A. C. Ferrari, Nat. Photonics, 2010, 4, 611-622.
20 N. V. R. Nulakani, M. Kamaraj and V. Subramanian, RSC Adv., 2015, 5, 78910-78916.

21 K. F. Mak, C. Lee, J. Hone, J. Shan and T. F. Heinz, Phys. Rev. Lett., 2010, $\mathbf{1 0 5}, 136805$.

22 H. Wang, H. Yuan, S. S. Hong, Y. Li and Y. Cui, Chem. Soc. Rev., 2015, 44, 2664-2680.

23 F. Zhang, Z. Wu, Z. Wang, D. Wang, S. Wang and X. Xu, RSC Adv., 2016, 6, 20027-20033

24 D. Q. Fang, S. L. Zhang and H. Xu, RSC Adv., 2013, 3, 24075-24080.

25 F. Xia, H. Wang, D. Xiao, M. Dubey and A. Ramasubramaniam, Nat. Photonics, 2014, 8, 899-907.

26 X. Li, J. Zhu and B Wei, Chem. Soc. Rev., 2016, Advance Article. DOI: $10.1039 / \mathrm{c} 6 \mathrm{cs} 00195 \mathrm{e}$

27 D. M. Schaadt, B. Feng and E. T. Yu, Appl. Phys. Lett., 2005, 86, 063106.

28 N. Liu, M. Mesch, T. Weiss, M. Hentschel and H. Giessen, Nano Lett., 2010, 10, 2342-2348.

29 H. Choi, S. J. Ko, Y. Choi, P. Joo, T. Kim, B. R. Lee, J. W. Jung, H. J. Choi, M. Cha, J. R. Jeong, I. W. Hwang, M. H. Song, B. S. Kim and J. Y. Kim, Nat. Photonics, 2013, 7, 732-738.

30 C. D. Bohn, A. Agrawal, Y. Lee, C. J. Choi, M. S. Davis, P. M. Haney, H. J. Lezec and V. A. Szala, Phys. Chem. Chem. Phys., 2014, 16, 6084-6091.

31 J. L. Perchec, Y. Desieres and R. Espiau de Lamaestre, Appl. Phys. Lett., 2009, 94, 181104.

32 S. Song, Q. Chen, L. Jin and F. Sun, Nanoscale, 2013, 5, 9615-9619.

33 Y. Cai, J. Zhu and Q. H. Liu, Appl. Phys. Lett., 2015, 106, 043105.

34 S. Heeg, R. Fernandez-Garcia, A. Oikonomou, F. Schedin, R. Narula, S. A. Maier, A. Vijayaraghavan and S. Reich, Nano Lett., 2012, 13, 301-308.

35 F. Wang, Q. Wang, G. Xu, R. Hui and J. Wu, J. Phys. Chem. C, 2013, 117, 11725-11730.

36 V. V. Khardikov, E. O. Iarko and S. L. Prosvirnin, J. Opt., 2010, 12, 045102.

37 H. Lu, B. P. Cumming and M. Gu, Opt. Lett., 2015, 40, 3647-3650.

38 B. Vasic and R. Gajic, Appl. Phys. Lett., 2013, 103, 261111.

39 X. Dang, J. Qi, M. T. Klug, P. Y. Chen, D. S. Yun, N. X. Fang, P. T. Hammond and A. M. Belcher, Nano Lett., 2013, 13, 637-642.

40 F. Xiong, J. Zhang, Z. Zhu, X. Yuan and S. Qin, Sci. Rep., 2015, 5, 16998.

41 A. H. Castro Neto, F. Guinea, N. M. R. Peres, K. S. Novoselov and A. K. Geim, Rev. Mod. Phys., 2009, 81, 109.

42 A. K. Geim, Science, 2009, 324, 1530-1534.

43 K. S. Novoselov, V. I. Falko, L. Colombo, P. R. Gellert, M. G. Schwab and K. Kim, Nature, 2012, 490, 192-200.

44 H. C. Chung, C. P. Chang, C. Y. Lin and M. F. Lin, Phys. Chem. Chem. Phys., 2016, 18, 7573-7616.

45 A. N. Grigorenko, M. Polini and K. S. Novoselov, Nat. Photonics, 2012, 6, 749-758.

46 V. W. Brar, M. S. Jang, M. Sherrott, J. J. Lopez, and H. A. Atwater, Nano Lett., 2013, 13, 2541-2547.

47 M. S. Jang, V. W. Brar, M. Sherrott, J. J. Lopez, L. Kim, S. Kim, M. Choi and H. A. Atwater, Phys. Rev. B, 2014, 90, 165409.

48 X. He, Carbon, 2015, 82, 229-237.

49 X. He, C. Liu, X. Zhong and W. Shi, RSC Adv., 2015, 5, 11818-11824.

50 Q. Lin, X. Zhai, L. Wang, B. Wang, G. Liu and S. Xia, EPL, 2015, 111, 34004.

51 X. Han, T. Wang, X. Li, S. Xiao and Y. Zhu, Opt. Express, 2015, 23, 31945-31955.

52 G. D. Liu, X. Zhai, L. L. Wang, B. X. Wang, Q. Lin and X. J. Shang, Plasmonics, 2016, 11, 381-387.

53 Z. Fang, Z. Liu, Y. Wang, P. M. Ajayan, P. Nordlander and N. J. Halas, Nano Lett., 2012, 12, 3808-3813.

54 A. Y. Nikitin, F. Guinea, F. J. Garcia-Vidal, L. Martin-Moreno, Phys. Rev. $B, 2012, \mathbf{8 5}, 081405$. 
55 S. Ke, B. Wang, H. Huang, H. Long, K. Wang and P. Lu, Opt. Express, 2015, 23, 8888-8900.

56 B. Xu, C. Gu, Z. Li and Z. Niu, Opt. Express, 2013, 21, 23803-23811.

57 Z. Liu, P. Zhan, J. Chen, C. Tang, Z. Yan, Z. Chen and Z. Wang, Opt. Express, 2013, 21, 3021-3030.

58 Y. Zhang, Y. Feng, B. Zhu, J. Zhao and T. Jiang, Opt. Express, 2014, 22, 22743-22752.

59 G. Yao, F. Ling, J. Yue, C. Luo, J. Ji and J. Yao, Opt. Express, 2016, 24, 1518-1527.

60 P. Liu, W. Cai, L. Wang, X. Zhang and J. Xu, Appl. Phys. Lett., 2012, 100, 153111.

61 K. Li, X. Ma, Z. Zhang, J. Song, Y. Xu and G. Song, J. Phys. D: Appl. Phys., 2014, 47, 405101.

62 E. D. Palik, Handbook of optical constants of solids, Academic Press, Boston, 1991, vol. 2.

63 W. Xu, Z. H. Zhu, K. Liu, J. F. Zhang, X. D. Yuan, Q. S. Lu and S. Q. Qin, Opt. Lett., 2015, 40, 1603-1606.

64 J. Zhang, Z. Zhu, W. Liu, X. Yuan and S. Qin, Nanoscale, 2015, 7, 1353013536.

65 J. Zhang, C. Guo, K. Liu, Z. Zhu, W. Ye, X. Yuan and S. Qin, Opt. Express, 2014, 22, 12524-12532.

66 A. Vakil and N. Engheta, Science, 2011, 332, 1291-1294.

67 S. Thongrattanasiri, I. Silveiro and F. J. Garcia de Abajo, Appl. Phys. Lett., 2012, 100, 201105.

68 K. Li, X. Ma, Z. Zhang and G. Song, J. Appl. Phys., 2014, 115, 104306. 69 S. Bae, H. Kim, Y. Lee, X. Xu, J. S. Park, Y. Zheng, J. Balakrishnan, T.
Lei, H. R. Kim, Y. I. Song, Y. J. Kim, K. S. Kim, B. Ozyilmaz, J. H. Ahn, B. H. Hong and S. Iijima, Nat. Nanotechnol., 2010, 5, 574-578.

70 H. Yan, X. Li, B. Chandra, G. Tulevski, Y. Wu, M. Freitag, W. Zhu, P. Avouris and F. Xia, Nat. Nanotechnol., 2012, 7, 330-334.

71 K. S. Novoselov, A. K. Geim, S. V. Morozov, D. Jiang, M. I. Katsnelson, I. V. Grigorieva, S. V. Dubonos and A. A. Firsov, Nature, 2005, 438, $197-$ 200.

72 Y. Zhang, Y. W. Tan, H. L. Stormer and P. Kim, Nature, 2005, 438, 201204.

73 A. Splendiani, L. Sun, Y. Zhang, T. Li, J. Kim, C. Y. Chim, G. Galli and Feng Wang, Nano Lett. 2010, 10, 1271-1275.

74 M. Liu, X. Yin, E. Ulin-Avila, B. Geng, T. Zentgraf, L. Ju, F. Wang and Xiang Zhang, Nature 2011, 474, 64-67.

75 X. Gan, R. J. Shiue, Y. Gao, I. Meric, T. F. Heinz, K. Shepard, J. Hone, S. Assefa and Dirk Englund, Nat. Photonics, 2013, 7, 883-887.

76 X. Gan, K. F. Mak, Y. Gao, Y. You, F. Hatami, J. Hone, T. F. Heinz and D. Englund, Nano Lett., 2012, 12, 5626-5631.

77 X. Gan, Y. Gao, K. F. Mak, X. Yao, R. J. Shiue, A. van. der. Zande, M. E. Trusheim, F. Hatami, T. F. Heinz, J. Hone and D. Englund, Appl. Phys. Lett., 2013, 103, 181119.

78 O. Lopez-Sanchez, D. Lembke, M. Kayci, A. Radenovic and A. Kis, Nat. Nanotechnol., 2013, 8, 497-501.

79 W. Wang, A. Klots, D. Prasai, Y. Yang, K. I. Bolotin and J. Valentine, Nano Lett., 2015, 15, 7440-7444.

80 S. Thongrattanasiri, F. H. L. Koppens and F. J. G. de Abajo, Phys. Rev. Lett., 2012, 108, 047401. 


\title{
Electronic Supplementary Information - Tunable light trapping and absorption enhancement with graphene ring arrays
}

\author{
Shuyuan Xiao, ${ }^{a}$ Tao Wang, ${ }^{*} a$ Yuebo Liu, ${ }^{b}$ Chen Xu, ${ }^{c}$ Xu Han, ${ }^{a}$ and Xicheng $\operatorname{Yan}^{a}$
}

The light trapping and absorption enhancement could also be tuned with the geometric variations of the structure in addition to manipulating the Fermi energy of graphene. In Figure S1 (a) and (b), the dependences of absorption in the absorbing layer on the radius and the width of the graphene ring are respectively presented. As the radius increases from $R=65 \mathrm{~nm}$ to $R=85 \mathrm{~nm}$, the resonance undergoes a redshift from $16.7 \mu \mathrm{m}$ to $21.8 \mu \mathrm{m}$, which agrees with the prediction from the equation (5). Meanwhile the absorption in the absorbing layer increases from $A^{\prime}=16.4 \%$ to $A^{\prime}=18.4 \%$ due to the increasing filling factor of graphene, and for the same reason, the similar increment in absorption from $A^{\prime}=15.6 \%$ to $A^{\prime}=18.3 \%$ can be observed with the increase of the width from $W=25 \mathrm{~nm}$ to $W=35 \mathrm{~nm}$.

The basic idea of the perfect light absorption is to minimize the reflection through impedance matching and simultaneously eliminate the transmission by maximizing the losses. To these ends, a newly added insulating layer and a gold mirror are introduced at the bottom of the original structure, as schematically shown in Figure S2 (a). The thickness of this insulating layer is set to $t_{i}^{\prime}=3 \mu \mathrm{m}$ and the permittivity is $\varepsilon_{d}=1.96$, while the gold mirror is treated as 200-nm-thick film and the permittivity is described by the Drude model with the plasma frequency $\omega_{p l}=1.37 \times 10^{16} s^{-1}$ and the damping constant $\omega_{c}=1.23 \times 10^{14} s^{-1}$, which is three times larger than the bulk value due to the surface scattering and grain boundary effects in thin films. In Figure S2 (b), perfect light absorption (99\%) in the hybrid structure is obtained at $20.0 \mu \mathrm{m}$, where the absorption in the absorbing layer reaches as high as $55.2 \%$ with an enhancement factor of 27.6 .

\footnotetext{
${ }^{a}$ Wuhan National Laboratory for Optoelectronics, Huazhong University of Science and Technology, Wuhan 430074, People's Republic of China. E-mail: wangtao@hust.edu.cn

${ }^{b}$ School of Information and Optoelectronic Science and Engineering, South China Normal University, Guangzhou 510006, People's Republic of China

${ }^{c}$ Department of Physics, New Mexico State University, Las Cruces 88001, United State of America
} 


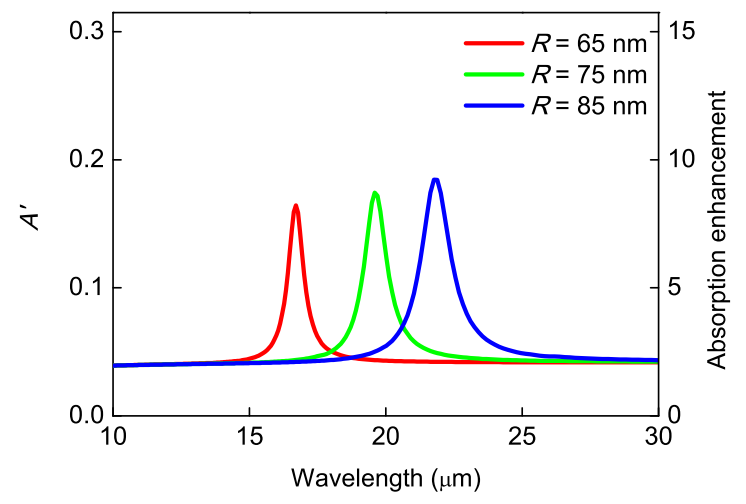

(a)

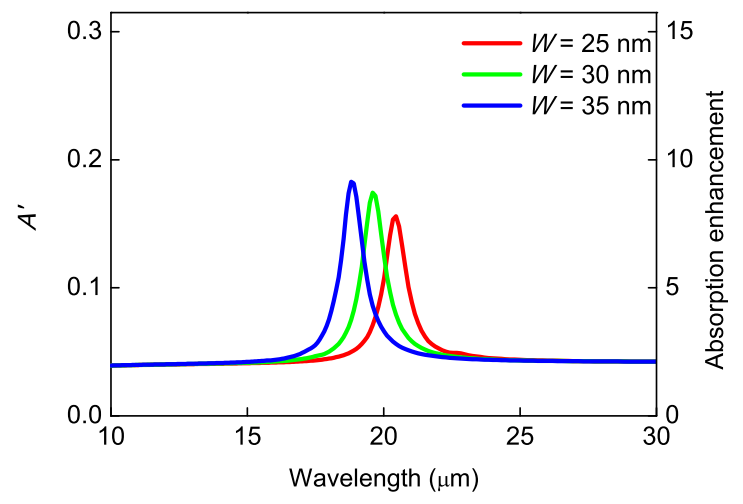

(b)

Figure S1: The simulated absorption in the absorbing layer $A^{\prime}$ with the attenuation coefficient $\alpha=-0.1 \mu \mathrm{m}^{-1}$, the Fermi energy of graphene $E_{F}=0.6 \mathrm{eV}$ and (a) the radius of graphene ring $R$ ranging from 65 to $85 \mathrm{~nm}$ and (b) the width of the graphene ring $W$ ranging from 25 to $35 \mathrm{~nm}$. The enhancement factor of absorption in the absorbing layer is also shown compared to that in the impedance matched media.

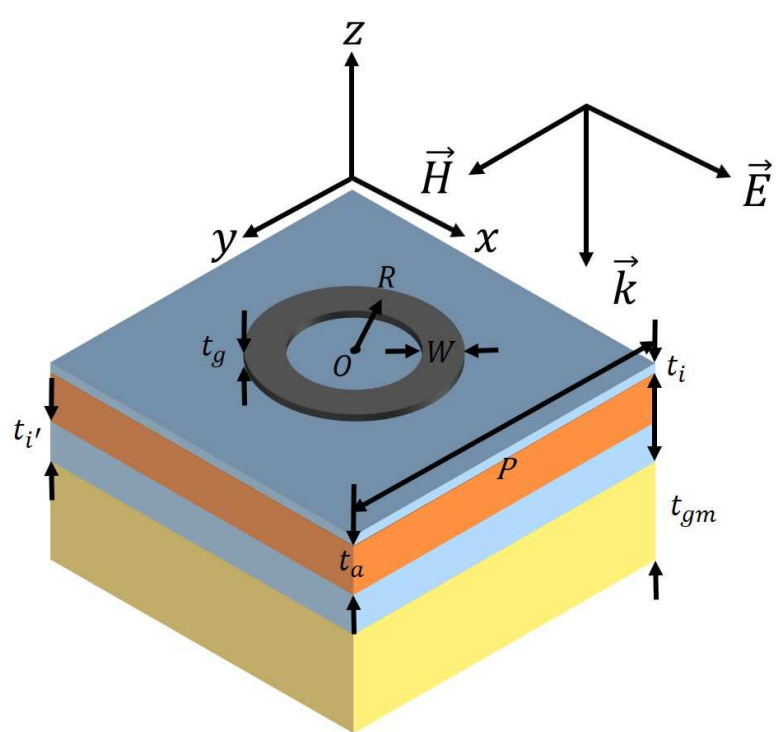

(a)

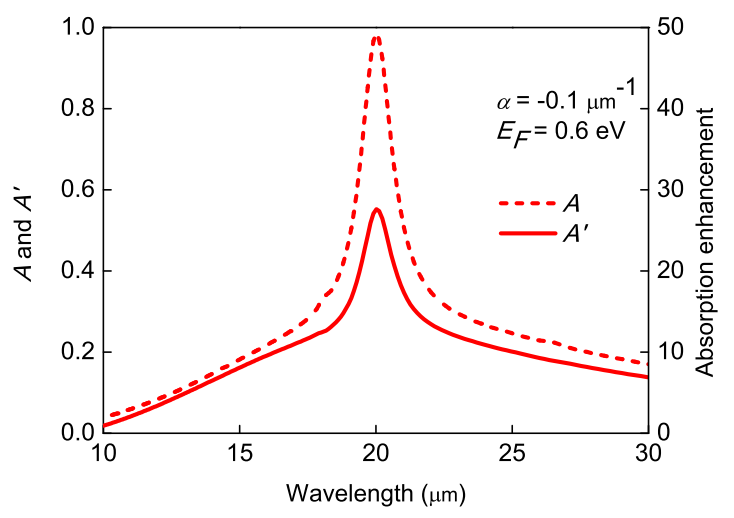

(b)

Figure S2: Perfect light absorption in the hybrid structure and further absorption enhancement in the absorbing layer with a gold mirror. (a) The schematic geometry of the unit cell backed by a gold mirror and (b) the simulated absorption $A$ and the absorption in the absorbing layer $A^{\prime}$ with the attenuation coefficient $\alpha=-0.1$ $\mu \mathrm{m}^{-1}$ and the Fermi energy of graphene $E_{F}=0.6 \mathrm{eV}$. The enhancement factor of absorption in the absorbing layer is also shown compared to that in the impedance matched media. 